\title{
Mislocated extrauterine intrauterine devices: Diagnosis and surgical management
}

\author{
Mustafa Kaplanoğlu ${ }^{1}$, Mehmet Bülbül ${ }^{1}$, Tuncay Yüce ${ }^{2}$, Dilek Kaplanoğlu ${ }^{1}$, Meral Aban ${ }^{3}$ \\ 'Department of Obstetric and Gynecology, Adiyaman University Faculty of Medicine, Adiyaman, Turkey \\ 2Department of Obstetric and Gynecology, Ankara University Faculty of Medicine, Ankara, Turkey \\ ${ }^{3}$ Department of Obstetric and Gynecology, Division of Gynecologic Oncology, Kolan International Hospital, istanbul, Turkey
}

Abstract

Objective: Presentation of the diagnostic and surgical treatment methods of our extrauterine intrauterine device (IUD) cases.

Material and Methods: We retrospectively evaluated the data of 21 extrauterine IUD cases at our clinic between 2008 and 2010 . The symptoms, diagnostic methods, and surgical treatments were evaluated.

Results: A total of 14 copper and seven levonorgestrel (LNG) IUDs were used. IUD had been inserted during lactation in $71.4 \%$ of the patients. The reasons for presentation of patients were unintended pregnancy in $19.05 \%$, pelvic pain in 19.05\%, and pelvic pain with vaginal bleeding in $23.8 \%$. IUD in two patients were located the retroperitoneal area. IUD string had not been visible during routine follow-up in $38.1 \%$ of the patients. Laparoscopy was performed in 14 patients and laparotomy was performed for dense adhesions in seven patients.

Conclusion: Extrauterine IUDs can present with various clinical symptoms. Ultrasonography and X-Ray are sufficient for the diagnosis. Surgical removal is needed to prevent possible complications, and the preferred surgical technique in appropriate patients is laparoscopy.

(J Turk Ger Gynecol Assoc 2015; 16: 91-5)

Keywords: Extrauterine IUD, surgery, contraception

Received: 23 January, 2015

Accepted: 29 March, 2015

\section{Introduction}

Intrauterine devices (IUDs) are highly effective reversible contraceptive devices commonly used throughout the world. Globally, they are the second most common contraceptive method (14\%) following sterilization (21\%) (1). They are also frequently used in Turkey (20.2\%). The most common types are the copper-releasing IUD and the Levonorgestrelreleasing IUD (LNG-IUD) systems.

There are various complications with respect to IUD use (0.2$3.6 / 1000$ applications), among which uterine perforation is a serious complication. Most uterine perforations are observed in IUD's inserted during lactation $(2,3)$. Other important complications are lower midline abdominal pain, organ perforation, strangulation, infection, and infertility. The adhesions that can gradually develop with intra-abdominal IUD's are the most important cause of additional morbidity (4). This complication has been reported both with LNG-IUDs (Mirena, Shering Plough, Germany) and copper IUDs (TCu-380A). Prevention and early diagnosis are the most important factors to avoid this complication. Therefore, it is necessary to see the IUD string immediately after insertion or in the first week with vaginal examination and to perform a check with ultrasonography in all patients. Surgical intervention is frequently required in uterine perforation.
We aim to present our clinical experience with the symptoms, diagnostic methods, and surgical treatment of extrauterine IUDs in this retrospective study.

\section{Material and Methods}

We retrospectively evaluated the patient charts of females with an extrauterine IUD and who had undergone laparotomy or laparoscopy at Adıyaman University Faculty of Medicine Training and Research Hospital, Department of Obstetrics and Gynecology between 2008 and 2014.

We first performed a search using the "IUD with no visible string at the cervical os" code (530.583) of the International Classification of Disease and Related Health Problems-10 (ICD-10) codes that have been adapted to Turkish. Following the evaluation of the data, the patients were divided into two groups as intrauterine and extrauterine IUDs. Patients who had an intrauterine IUD where no string was visible and that was removed with a simple vaginal procedure or hysteroscopy were excluded from the study. We found a total of 21 extrauterine IUD cases. Patient data were evaluated through review of the patient charts and the hospital system records. All patients underwent surgery after going through a similar evaluation process. A gynecological examination was performed first and IUD was evaluated with transvaginal ultra- 
Table 1. Demographic characteristics of patients

\begin{tabular}{|c|c|c|c|c|c|c|c|c|}
\hline Patient & $\begin{array}{c}\text { Age } \\
\text { (years) }\end{array}$ & Gravity & Parity & $\underset{\left(\mathbf{k g} / \mathbf{m}^{2}\right)}{\mathrm{BMI}}$ & $\begin{array}{c}\text { Number of } \\
\text { CS }\end{array}$ & $\begin{array}{c}\text { Previous IUD } \\
\text { use }\end{array}$ & $\begin{array}{l}\text { Time of total } \\
\text { IUD use } \\
\text { (months) }\end{array}$ & $\begin{array}{c}\text { History of } \\
\text { abdomina } \\
\text { surgery }\end{array}$ \\
\hline 1 & 23 & 3 & 3 & 26.4 & None & yes & 24 & no \\
\hline 2 & 25 & 3 & 3 & 23.6 & 1 & no & 0 & no \\
\hline 3 & 28 & 4 & 3 & 27.8 & None & yes & 48 & no \\
\hline 4 & 26 & 2 & 2 & 26.4 & None & no & 0 & no \\
\hline 5 & 24 & 1 & 1 & 29.1 & None & no & 0 & yes \\
\hline 6 & 34 & 5 & 5 & 28 & 1 & no & 0 & no \\
\hline 7 & 38 & 7 & 4 & 26.1 & None & no & 0 & no \\
\hline 8 & 36 & 5 & 4 & 26.5 & 3 & no & 0 & no \\
\hline 9 & 34 & 3 & 3 & 25.9 & None & no & 0 & no \\
\hline 10 & 31 & 4 & 4 & 24.9 & 2 & yes & 30 & no \\
\hline 11 & 30 & 2 & 2 & 24.7 & None & yes & 48 & yes \\
\hline 12 & 23 & 2 & 2 & 28.6 & None & no & 0 & no \\
\hline 13 & 27 & 1 & 1 & 27 & None & no & 0 & no \\
\hline 14 & 28 & 2 & 2 & 26.2 & None & no & 0 & no \\
\hline 15 & 35 & 6 & 4 & 29 & None & yes & 48 & no \\
\hline 16 & 30 & 4 & 4 & 26 & None & no & 0 & no \\
\hline 17 & 26 & 3 & 3 & 31 & None & no & 0 & no \\
\hline 18 & 32 & 8 & 6 & 27 & None & yes & 36 & yes \\
\hline 19 & 36 & 5 & 4 & 28 & None & yes & 48 & no \\
\hline 20 & 28 & 5 & 3 & 24 & 1 & no & 0 & no \\
\hline 21 & 32 & 3 & 3 & 25.4 & None & yes & 36 & no \\
\hline
\end{tabular}

sonography when the string could not be seen. Anteroposterior and lateral pelvic X-rays were obtained to support the diagnosis. None of the patients in the study underwent magnetic resonance imaging (MRI) or computed tomography (CT) scans. Patients who received a preliminary diagnosis of extrauterine IUD were prepared for laparoscopic surgery. Laparotomy with a Pfannenstiel incision was performed for patients who could not undergo laparoscopy. All surgical procedures were performed under general anesthesia.

The 21 patients detected within the defined period were evaluated. Age, obstetric history, extrauterine IUD type, time of application, time until diagnosis and surgical intervention, symptoms, and surgical route used were recorded. Informed written consent was obtained from all the women. This retrospective case-series study was exempt from ethical approval by the Adiyaman University Ethics commission. We did not conduct statistical analysis.

\section{Results}

\section{Demographic data}

The demographic data revealed a mean age of 29.4 years and a mean body mass index (BMI) value of $26.9 \mathrm{~kg} / \mathrm{m}^{2}$. Obstetric data revealed a mean gravida value of 3.6. The mean number of births by normal spontaneous vaginal delivery was 2.5 , while the mean number of cesarean births was 0.4. An IUD had been used before the current one by $33.3 \%$ of the patients. The mean duration for which the previous IUD was used was 40.2 (24-48) months (Table 1).

\section{IUD types and important time points regarding insertion}

Table 2 presents the extrauterine IUD clinical data and usage characteristics of the patients. All mislocated extrauterine IUD applications had been performed in primary health care centers. Extrauterine IUDs consisted of seven LNG-IUDs and 14 copper IUDs (TCu-380A-IUD). The mean duration between IUD insertion and diagnosis was 8.1 months (3 days-24 months). The mean duration between the last pregnancy and IUD insertion was 14.7 (2-38) months. IUD had been inserted during lactation in $15(71.4 \%)$ patients.

\section{Symptoms and IUD localization}

Symptoms at presentation were delayed menstruation in the four patients with an unintended pregnancy, pelvic pain in four, and pelvic pain with vaginal bleeding in five. In addition, the IUD strings of eight patients had not been visible during routine follow-up. Two of the patients with delayed menstruation had undergone pregnancy termination at private centers, while two 
Table 2. Symptoms, the locations and treatment of IUD, type of IUDs and special time for IUD insertion

\begin{tabular}{|c|c|c|c|c|c|c|}
\hline $\begin{array}{l}\text { Patients } \\
\text { no }\end{array}$ & Location of the IUD & Type of operation & $\begin{array}{l}\text { Time from } \\
\text { insertion } \\
\text { to diagnosis }\end{array}$ & $\begin{array}{l}\text { Time from } \\
\text { pregnancy } \\
\text { to insertion }\end{array}$ & Symptom(s) & Type of IUD \\
\hline 1 & In the Douglas pouch & Laparoscopy & 7 months & 16 months & None & LNG-IUD \\
\hline 2 & In the Douglas pouch & Laparoscopy & 12 months & 11 months & None & TCu 380 A-IUD \\
\hline 3 & Near the uterine artery & Laparoscopy & 6 months & 8 months & None & TCu 380 A-IUD \\
\hline 4 & Near the ovary & Laparotomy & 3 months & 4 months & Pelvic pain & TCu 380 A-IUD \\
\hline 5 & In the Douglas pouch & Laparoscopy & 18 months & 2 months & None & TCu 380 A-IUD \\
\hline 6 & In the Douglas pouch & Laparoscopy & 24 months & 8 months & None & TCu 380 A-IUD \\
\hline 7 & In the Douglas pouch & Laparoscopy & 12 months & 16 months & Unintended pregnancy & TCu 380 A-IUD \\
\hline 8 & Omentum & Laparotomy & 10 months & 24 months & Unintended pregnancy & LNG-IUD \\
\hline 9 & Omentum & Laparotomy & 6 months & 38 months & Unintended pregnancy & LNG-IUD \\
\hline 10 & Omentum & Laparotomy & 4 days & 36 months & Pain and bleeding & LNG-IUD \\
\hline 11 & In the Douglas pouch & Laparoscopy & 7 days & 24 months & Pain and bleeding & LNG-IUD \\
\hline 12 & In the Douglas pouch & Laparoscopy & 3 days & 10 months & Pelvic pain & TCu 380 A-IUD \\
\hline 13 & In the Douglas pouch & Laparoscopy & 5 days & 8 months & Pain and bleeding & TCu 380 A-IUD \\
\hline 14 & Retroperitoneum & Laparotomy & 6 days & 6 months & Pain and bleeding & TCu 380 A-IUD \\
\hline 15 & In the Douglas pouch & Laparoscopy & 3 days & 22 months & Pelvic pain & LNG-IUD \\
\hline 16 & Retroperitoneum & Laparotomy & 24 months & 18 months & Unintended pregnancy & TCu 380 A-IUD \\
\hline 17 & Neary the ovary & Laparoscopy & 7 months & 12 months & None & TCu 380 A-IUD \\
\hline 18 & In the Douglas pouch & Laparoscopy & 13 months & 2 months & None & TCu 380 A-IUD \\
\hline 19 & Omentum & Laparotomy & 8 months & 36 months & None & LNG-IUD \\
\hline 20 & Near the ovary & Laparoscopy & 6 months & 6 months & Pelvic pain & TCu 380 A-IUD \\
\hline 21 & In the Douglas pouch & Laparoscopy & 18 months & 2 months & Pain and bleeding & TCu 380 A-IUD \\
\hline
\end{tabular}

patients continued the pregnancy with an extrauterine IUD and had a normal spontaneous vaginal delivery. IUD was in the Douglas pouch and retroperitoneum in the patients who gave birth. IUD was surgically removed in the postpartum period in both patients. The retroperitoneal IUD was close to the right iliac artery bifurcation during the surgery. The surgery started as laparoscopy but was converted to laparotomy because of the IUD location and the dense adhesions.

The most common extrauterine IUD location was the Douglas pouch with 11 (52.3\%) patients (Figure 1, 2). The rare location of the retroperitoneum was seen in two of our patients (Figure 3 ). Laparoscopic IUD removal was performed in a total of 14 (66.6\%) patients. The most common reason to perform laparotomy was severe adhesions.

Table 2 presents the treatment method, IUD type, and localization. Sixteen patients were prepared for laparoscopy under general anesthesia. The surgery was converted to laparotomy because of dense adhesions in two patients. Laparotomy had been performed in five patients because of technical equipment failure. General surgery consultation was required for dense adhesions and abscess formation in two of the patients whose surgeries were converted to laparotomy. The patient with an abscess underwent abscess drainage, and there was no additional complication during follow-up. Both patients had a TCu-380A-IUD. There was no intraoperative complication.

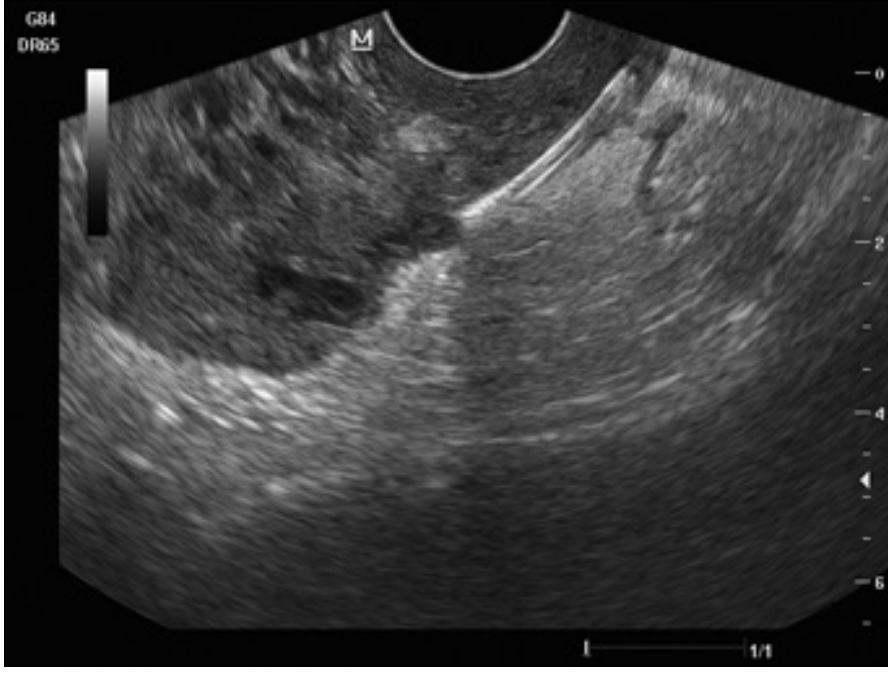

Figure 1. Ultrasonographic appearance of T-shaped IUD in Douglas pouch

\section{Discussion}

IUD use is a modern contraceptive use that is commonly employed throughout the world. The low cost, long duration of effectiveness, high efficacy, reversibility, lack of systemic side 


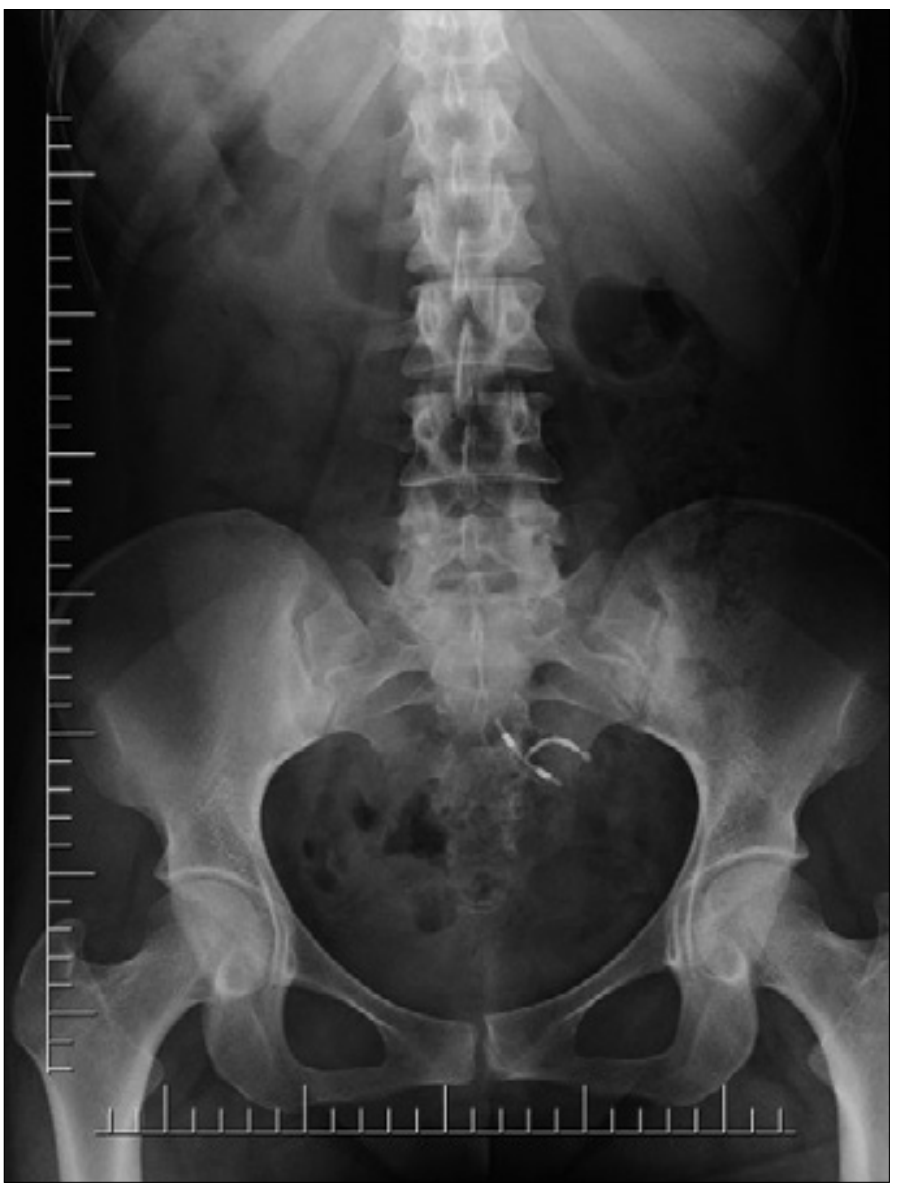

Figure 2. Anteroposterior abdominal X-ray demonstrating location of IUD (Coronal)

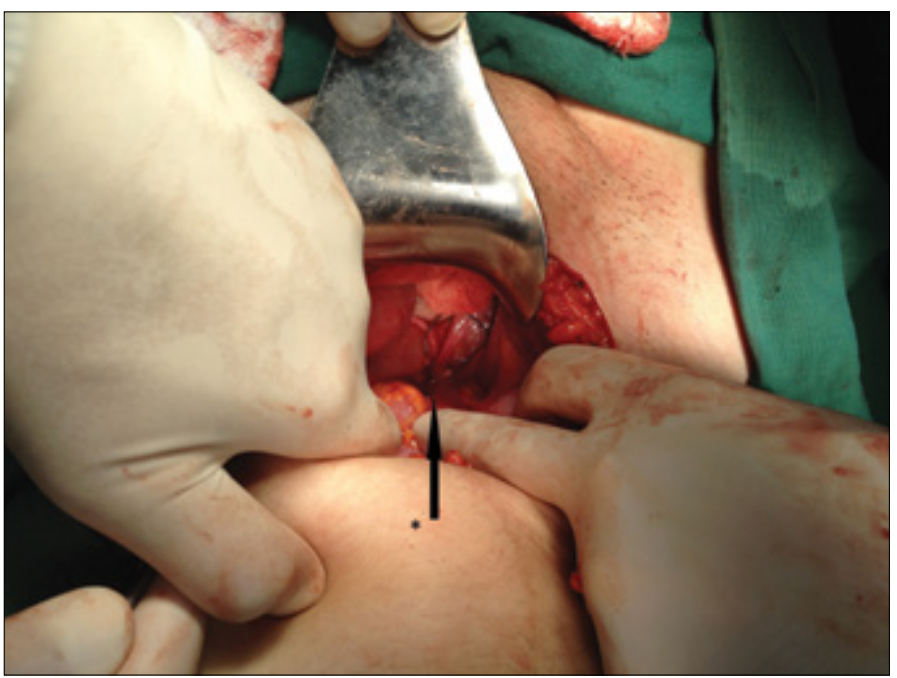

Figure 3. Intraoperative image of IUD (Laparotomy). The IUD string is intraperitoneal but the location of the IUD body is the retroperitoneal area

effects, and coitus-independent effectiveness have led to its widespread use. However, complications such as vaginal bleeding, abdominal pain, infection, uterus perforation, and migration to adjacent organs have been reported. Complications such as uterine perforation are rare but very well defined. Some risk factors for perforation with IUD use have been reported. The postpartum period, lactation period, uterus with undiagnosed pregnancy, congenital uterine abnormality, and excessive anteflexion or retroflexion are important risk factors. The most common association is uterus perforation with an IUD inserted during the lactation period where the risk increases 10 -fold (5). Uterine perforation can cause vaginal bleeding and lower abdominal pain following insertion, but several cases have no symptoms for an extended period.

Several studies have reported a period of $<1$ year between IUD insertion and uterine perforation diagnosis; at least $80 \%$ of these patients have been found to be in the lactation period at the time of insertion $(3,6)$. Similarly, $71.4 \%$ of our patients had been in the lactation period during IUD insertion. However, the diagnosis can be delayed by months or even years with an intraperitoneal IUD. The main step in the diagnosis is suspecting an extrauterine IUD in patients where the IUD string is not seen at the cervical os during routine follow-ups or normal outpatient visits. Transvaginal ultrasonography is the best method for localization in case of suspicion. The location may be the Douglas pouch, inside the broad ligament, attached to the omentum, or the retroperitoneal area. Lateral and anteroposterior pelvic X-rays can also be obtained to support the diagnosis and for additional information on the location.

For the treatment of extrauterine IUDs, the World Health Organization recommends prompt surgical removal after diagnosis, independent of the localization and symptoms (7). Prompt decision with regard to surgery is important because adhesion formation becomes more marked and removal becomes more difficult with extrauterine IUDs as time passes. However, there are contrasting views with regard to asymptomatic patients (8). The most important complications of intra-abdominal IUDs are adhesions, intestinal obstruction, chronic pelvic pain, abscess development, and infertility $(9,10)$. Therefore, it is particularly important to remove IUDs in symptomatic patients. The preferred surgical route is laparoscopy because it is minimally invasive and can be used with a high success rate (11). Laparotomy should only be used in patients with significant adhesion or adjacent organ invasion.

IUDs are inserted by midwives and medical practitioners following a certification program in our country. Therefore, early follow-ups can be a problem. The lack of suspicion about perforation in the early stage can delay referrals to a gynecology specialist, and therefore the diagnosis. However, LNG-IUDs are generally inserted by obstetrics and gynecology specialists in our country. We found an extrauterine LNG-IUD in seven patients. This result indicates that the uterine perforation risk should be considered even when the insertion has been performed by a specialist.

Operator factors were not addressed in our study, and this is one of the limitations of our research. We did not have access to information regarding who performed IUD procedures; therefore, we could not address operator skill as a factor for uterine perforation and malposition of IUD.

The migration of IUD to adjacent structures following perforation has been reported with extrauterine IUDs in the literature. 
Bladder and iliac vein migration is particularly noteworthy (12). Therefore, migration should be considered if IUD cannot be found (13-16). Retroperitoneal IUD migration is rare but important because of the adjacent vascular structures. Consultation of the relevant specialty may be intraoperatively acquired in such cases; we requested general surgery consultation because of diffuse intestinal adhesions for two cases and abscess formation at the sigmoid colon level for one case. Abscess drainage from the abdomen was performed for our patient with abscess formation. Most of the patients were diagnosed with an extrauterine IUD when the IUD string was not seen during routine follow-up and the patient was evaluated by ultrasonography and X-ray (17).

In conclusion, uterine perforation can present with symptoms such as lower abdominal pain and abnormal vaginal bleeding, with the IUD string not being seen during routine follow-up, or an unintended pregnancy. The combination of transvaginal ultrasound and anteroposterior/lateral X-ray is usually adequate for the diagnosis. It may be best for a specialist to perform the insertion in cases at risk such as those with a known uterine anomaly or excessive uterine anteflexion or retroflexion or patients in the lactation period. The early diagnosis of complications, such as uterine perforation, also requires the follow-up of the IUD location with postprocedure ultrasonography in several cases. In light of the data, we believe that all extrauterine IUD's should be surgically removed (preferably by laparoscopy) to prevent complications at later stages.

Ethics Committee Approval: Ethics committee approval was received for this study from the ethics committee of Adlyaman University Faculty of Medicine Ethical Committee.

Informed Consent: Written informed consent was obtained from patients who participated in this study.

Peer-review: Externally peer-reviewed.

Author Contributions: Concept - M.K., M.B., T.Y.; Design - M.K., M.B., T.Y.; Supervision - M.K., M.B., T.Y., MA; Resource - M.K., M.B., T.Y.; Materials - M.K., M.B., T.Y.; Data Collection \& /or Processing - M.K., M.B., T.Y., D.K.; Analysis \& /or Interpretation M.K., M.B., T.Y., D.K.; Literature Search - M.K., D.K., T.Y.; Writing - M.K., T.Y.; Critical Reviews - M.K., M.B., T.Y., D.K., M.A.

Conflict of Interest: No conflict of interest was declared by the authors.

Financial Disclosure: The authors declared that this study has received no financial support.

\section{References}

1. World Population Bureau. Family Planning Worldwide, 2008 Data Sheet. Available from: http://www.prb.org/pdf08/fpds08.pdf (29 January 2013, date last accessed)

2. Chi I, Feldblum PJ, Rogers SM. IUD-related uterine perforation: an epidemiologic analysis of a rare event using an international dataset. Contracept Deliv Syst 1984; 5: 123-30.

3. Caliskan E, Ozturk N, Dilbaz BO, Dilbaz S. Analysis of risk factors associated with uterine perforation by intrauterine devices. Eur J Contracept Reprod Health Care 2003; 8: 150-5. [CrossRef]

4. Adoni A, Ben Chetrit A. The management of intrauterine devices following uterine perforation. Contraception 1991; 43: 77-81. [CrossRef]

5. Heartwell SP, Schlesselman S. Risk of uterine perforation among users of intrauterine devices. Obstet Gynecol 1983; 61: 31-6.

6. Andersson K, Ryde-Blomqvist E, Lindell K, Odlind V, Milsom I. Perforations with intrauterine devices. Report from a Swedish survey. Contraception 1998; 57: 251-5. [CrossRef]

7. World Health Organization. Sexual and reproductive health. Available from: http://www.who.int/reproductivehealth/publications/maternal_perinatal_health/en/index.html

8. Markovitch O, Klein Z, Gidoni Y, Holzinger M, Beyth Y. Extrauterine mislocated IUD: is surgical removal mandatory? Contraception 2002; 66: 105-8. [CrossRef]

9. Rao RP. Lost intrauterine devices and their localization. J Reprod Med 1978; 20: 195-9.

10. Ohana E, Sheiner E, Leron E, Mazor M. Appendix perforation by an intrauterine contraceptive device. Eur J Obstet Gynecol Reprod Biol 2000; 88: 129-31. [CrossRef]

11. Demir SC, Cetin MT, Ucunsak IF, Atay Y, Toksoz L, Kadayifici O. Removal of intra-abdominal intrauterine device by laparoscopy. Eur J Contracept Reprod Health Care 2002; 1: 20-3. [CrossRef]

12. Roy KK, Banerjee N, Sinha A. Laparoscopic removal of translocated retroperitoneal IUD. Int J Gynaecol Obstet 2000; 71: 241-3. [CrossRef]

13. Farouk K, Afridi Z, Farooq M, Qureshi I. Urological complications of Intrauterine contraceptive device. Journal of Postgraduate Medical Institute 2007; 21: 260-5.

14. Yalcin V, Demirkesen O, Alici B, Onol B, Solok V. An unusual presentation of a foreign body in the urinary bladder: a migrant intrauterine device. Urol Int 1998; 61: 240-2. [CrossRef]

15. Demirci D, Ekmekcioglu O, Demirtas A, Gulmez I. Big bladder stones around an intravesical migrated intrauterine device. Int Urol Nephrol 2003; 35: 495-6. [CrossRef]

16. Kassab B, Audra P. The migrating intrauterine device. Case report and review of the literature. Contracept Fertil Sex 1999; 27: 696-700.

17. Ozgun MT, Batukan C, Serin IS, Ozcelik B, Basbug M, Dolanbay M. Surgical management of intra-abdominal mislocated intrauterine devices. Contraception 2007; 75: 96-100. [CrossRef] 